\title{
Dispersion of incoherent spectral features in systems with strong electron-phonon coupling
}

\author{
O. Röscha and O. Gunnarsson \\ Max-Planck-Institut für Festkörperforschung, Postfach 800665, D-70506 Stuttgart, Germany
}

Received: date / Revised version: date

\begin{abstract}
We study (inverse) photoemission from systems with strong coupling of doped carriers to phonons. Using an adiabatic approximation, we develop a method for calculating spectra. This method is particularly simple for systems where the electron-phonon coupling can be neglected in the initial state, e.g., the undoped $t-J$ model. The theory then naturally explains why the electron-phonon coupling just leads to a broadening of spectra calculated without electron-phonon coupling, without changing the dispersion. This is in agreement with recent angle-resolved photoemission spectroscopy (ARPES) on undoped cuprates, and it supports the interpretation in terms of strong electron-phonon interaction. The theory also shows that for systems with strong electron-phonon coupling in the initial state, the result cannot in general be related to the spectrum obtained without electron-phonon coupling.
\end{abstract}

PACS. 71.38.-k Polarons and electron-phonon interactions - 79.60.-i Photoemission and photoelectron spectra - 71.10.Fd Lattice fermion models

\section{Introduction}

Angle-resolved photoemission spectroscopy (ARPES) experiments have found evidence for strong electron-phonon interaction (EPI) and polaron physics in many materials like quasi-one-dimensional conductors $[1,2]$, the manganites [3], or the undoped high- $T_{c}$ cuprates [4-7]. The spectra show an incoherent broad feature whereas the quasiparticle peak at lower binding energy is strongly suppressed. In the case of the undoped high- $T_{c}$ cuprates, the dispersion of that broad peak matches the quasi-particle dispersion calculated in purely electronic models [6]. For an undoped $t-J$ model with coupling of doped holes to optical phonons, numerical calculations of the ARPES spectra indeed showed broad features tracing the dispersion of the quasi-particles in the original $t-J$ model [8]. Similar observations in the manganites [3] and quasi-onedimensional conductors $[1,2]$ have been interpreted in analogy with a single electron coupled to harmonic oscillators [9] and a related sum-rule for the first spectral moment. With respect to the manganites also the picture of the photohole seeing a frozen lattice has been used [10].

In this paper we address (inverse) photoemission spectra from systems with strong coupling of doped carriers to phonons. We develop a theory based on the adiabatic approximation. This theory takes a particularly simple form if the EPI can be neglected in the initial state. This is the case for the undoped $t-J$ model and the empty or full Holstein model. The spectrum can then be related to the

\footnotetext{
${ }^{a}$ e-mail: 0.Roesch@fkf.mpg.de
}

spectrum of a model without EPI, and the effect of the EPI is essentially to broaden the spectrum. For systems where the EPI is important also in the initial state, the theory takes a more complicated form. It can then be related to an average of spectra for distorted lattices without EPI.

After giving general arguments in section 2 we discuss numerical results for the Holstein model (section 3) and the $t$ - $J$ model with phonons (section 4 ) as illustrating examples.

\section{General considerations}

We consider a system that is modeled by the following Hamiltonian:

$$
H=H_{e l}+H_{p h}+H_{e p}
$$

$H_{e l}\left(H_{p h}\right)$ describes the purely electronic (phononic) part of the model whereas the interaction between electrons and phonons is given by $H_{e p}$.

The phonons are assumed to be harmonic in the absence of EPI and the system is taken to be translationally invariant, so that we can write:

$$
H_{p h}=\sum_{\mathbf{q}, \nu} \frac{1}{2}\left(\Pi_{\mathbf{q}, \nu} \Pi_{-\mathbf{q}, \nu}+\omega_{\mathbf{q}, \nu}^{2} Q_{\mathbf{q}, \nu} Q_{-\mathbf{q}, \nu}\right) .
$$

Here, an individual phonon mode with frequency $\omega_{\mathbf{q}, \nu}$ has wavevector $\mathbf{q}$ and belongs to branch $\nu$. Its generalized coordinate and momentum are denoted by $Q_{\mathbf{q}, \nu}$ and $\Pi_{\mathbf{q}, \nu}$. 
The EPI couples electronic degrees of freedom to the phonon coordinates $Q_{\mathbf{q}, \nu}$. We assume that this interaction vanishes for a certain electronic filling of the system which we will refer to as undoped in the following. The completely empty or completely filled Holstein or Holstein- $t-J$ model are examples for such undoped systems, see sections 3 and 4.

In addition, we assume that the phonon frequencies are small compared to the electronic energy scales defined by $H_{e l}$. This justifies an adiabatic approximation [11] and we can first consider only $H_{e l}+H_{e p}$ treating the phonon coordinates $Q_{\mathbf{q}, \nu}$ in $H_{e p}$ as c-numbers, i.e. as instantaneous parameters for the electronic problem. We denote the corresponding eigenstates and eigenvalues by $\left|E_{m}^{N_{e}}(\mathbf{Q})\right\rangle$ and $E_{m}^{N_{e}}(\mathbf{Q})$ which are labeled by the number of electrons $N_{e}$ and other quantum numbers $m$. We use $\mathbf{Q}$ as a shorthand notation for the set of phonon coordinates. The phonon eigenfunctions are then obtained by solving

$$
\left(\sum_{\mathbf{q}, \nu} \frac{1}{2} \Pi_{\mathbf{q}, \nu} \Pi_{-\mathbf{q}, \nu}+V_{m}^{N_{e}}(\mathbf{Q})\right) \phi_{m n}^{N_{e}}(\mathbf{Q})=\varepsilon_{m n}^{N_{e}} \phi_{m n}^{N_{e}}(\mathbf{Q})
$$

a Schrödinger equation with the effective potential

$$
V_{m}^{N_{e}}(\mathbf{Q})=E_{m}^{N_{e}}(\mathbf{Q})+\sum_{\mathbf{q}, \nu} \frac{1}{2} \omega_{\mathbf{q}, \nu}^{2} Q_{\mathbf{q}, \nu} Q_{-\mathbf{q}, \nu}
$$

The eigenenergies are $\varepsilon_{m n}^{N_{e}}$ where $n$ stands for suitable phonon quantum numbers. In our approximation the eigenstates of $H$ are $\left\langle\mathbf{Q} \mid \varepsilon_{m n}^{N_{e}}\right\rangle=\phi_{m n}^{N_{e}}(\mathbf{Q})\left|E_{m}^{N_{e}}(\mathbf{Q})\right\rangle$, i.e. we assume that the electronic states $\left|E_{m}^{N_{e}}(\mathbf{Q})\right\rangle$ do not mix.

The ground-state in a system with $N_{e}$ electrons is then given by $\left\langle\mathbf{Q} \mid \varepsilon_{00}^{N_{e}}\right\rangle=\phi_{00}^{N_{e}}(\mathbf{Q})\left|E_{0}^{N_{e}}(\mathbf{Q})\right\rangle$ with eigenenergy $\varepsilon_{00}^{N_{e}}$. In case of an undoped system with $N_{e}^{0}$ electrons there is no EPI and the phonon wavefunction just corresponds to the ground-state of $H_{p h}$ :

$$
\phi_{00}^{N_{e}^{0}}(\mathbf{Q})=\prod_{\mathbf{q} \nu}\left(\omega_{\mathbf{q}, \nu} / \pi\right)^{1 / 4} \exp \left(-\omega_{\mathbf{q}, \nu} Q_{\mathbf{q}, \nu}^{2} / 2\right) .
$$

The electronic ground-state and its eigenenergy are then independent of $\mathbf{Q}$, and $\varepsilon_{00}^{N_{e}^{0}}=E_{0}^{N_{e}^{0}}+\sum_{\mathbf{q}, \nu} \omega_{\mathbf{q}, \nu} / 2$.

We now do (inverse) photoemission at zero temperature by destroying (creating) an electron with momentum $\mathbf{k}$ and spin $\sigma$ in the ground-state of a system with $N_{e}$ electrons. Within the adiabatic approximation this can be described by considering the following Green's function

$$
\begin{aligned}
G_{\mathbf{k}, \sigma}^{N_{e}, \mp}(z)= & \left\langle\varepsilon_{00}^{N_{e}}\left|\psi^{\dagger} \frac{1}{z-\left(H-\varepsilon_{00}^{N_{e}}\right)} \psi\right| \varepsilon_{00}^{N_{e}}\right\rangle \\
= & \int d \mathbf{Q} \int d \mathbf{Q}^{\prime} \phi_{00}^{N_{e} *}(\mathbf{Q})\left\langle E_{0}^{N_{e}}(\mathbf{Q})\right| \psi^{\dagger} \times \\
& \times\left\langle\mathbf{Q}\left|\frac{1}{z-\left(H-\varepsilon_{00}^{N_{e}}\right)}\right| \mathbf{Q}^{\prime}\right\rangle \psi\left|E_{0}^{N_{e}}\left(\mathbf{Q}^{\prime}\right)\right\rangle \phi_{00}^{N_{e}}\left(\mathbf{Q}^{\prime}\right)
\end{aligned}
$$

where $\psi=c_{\mathbf{k}, \sigma}^{(\dagger)}$ and $\int d \mathbf{Q}=\prod_{\mathbf{q}, \nu} \int d Q_{\mathbf{q}, \nu}$.

We proceed in analogy to Ref. [12] and neglect the kinetic energy of the phonons in the resolvent in Eq. (6).
Now $H$ is diagonal in the phonon coordinates $\mathbf{Q}$ and one half of the integrations in Eq. (6) can be eliminated. This leads to the following approximation for the Green's function [12]:

$$
\tilde{G}_{\mathbf{k}, \sigma}^{N_{e}, \mp}(z)=\int d \mathbf{Q}\left|\phi_{00}^{N_{e}}(\mathbf{Q})\right|^{2} g_{\mathbf{k}, \sigma}^{N_{e}, \mp}\left(\tilde{z}_{\mathbf{Q}}, \mathbf{Q}\right)
$$

where

$g_{\mathbf{k}, \sigma}^{N_{e}, \mp}\left(\tilde{z}_{\mathbf{Q}}, \mathbf{Q}\right)=\left\langle E_{0}^{N_{e}}(\mathbf{Q})\left|\psi^{\dagger} \frac{1}{\tilde{z}_{\mathbf{Q}}-\left(H(\mathbf{Q})-\varepsilon_{00}^{N_{e}}\right)} \psi\right| E_{0}^{N_{e}}(\mathbf{Q})\right\rangle$.

where

$$
\tilde{z}_{\mathbf{Q}}=z+V_{0}^{N_{e}}(\mathbf{Q})-\varepsilon_{00}^{N_{e}} .
$$

Finally, the corresponding spectral function is given by

$$
\begin{aligned}
\tilde{A}_{\mathbf{k}, \sigma}^{N_{e}, \mp}(\omega) & =\frac{1}{\pi} \operatorname{Im} \tilde{G}_{\mathbf{k}, \sigma}^{N_{e}, \mp}\left(\omega-i 0^{+}\right) \\
& =\int d \mathbf{Q}\left|\phi_{00}^{N_{e}}(\mathbf{Q})\right|^{2} \rho_{\mathbf{k}, \sigma}^{N_{e}, \mp}(\omega, \mathbf{Q})
\end{aligned}
$$

where

$$
\begin{aligned}
\rho_{\mathbf{k}, \sigma}^{N_{e}, \mp}(\omega, \mathbf{Q})= & \sum_{m}\left|\left\langle E_{m}^{N_{e} \mp 1}(\mathbf{Q})|\psi| E_{0}^{N_{e}}(\mathbf{Q})\right\rangle\right|^{2} \times \\
& \times \delta\left(\omega-\left(V_{m}^{N_{e} \mp 1}(\mathbf{Q})-V_{0}^{N_{e}}(\mathbf{Q})\right)\right)
\end{aligned}
$$

after expanding $\psi\left|E_{0}^{N_{e}}(\mathbf{Q})\right\rangle$ in the adiabatic electronic basis states $\left|E_{m}^{N_{e} \mp 1}(\mathbf{Q})\right\rangle$.

Equations (9-10) will turn out to be the key formula for interpreting ARPES spectra of undoped systems. To see this we observe that $\rho_{\mathbf{k}, \sigma}^{N_{e}, \mp}(\omega, \mathbf{Q})$ is the spectral function of the system without EPI for a given lattice distortion $\mathbf{Q}$. If we assume that $V_{0}^{N_{e}}(\mathbf{Q})$ has a non-degenerate absolute minimum at $\mathbf{Q}_{\min }$ the corresponding ground-state phonon wave-function will be localized around this point in coordinate space. If we approximate $\left|\phi_{00}^{N_{e}}(\mathbf{Q})\right|^{2} \approx \delta\left(\mathbf{Q}-\mathbf{Q}_{\text {min }}\right)$ we find that the spectrum corresponds to the spectrum one obtains for the system with a frozen distortion $\mathbf{Q}_{\min }$ in which there is no EPI. Analogously, in case of more than one (quasi-)degenerate minima of $V_{0}^{N_{e}}(\mathbf{Q})$ we have to take the (weighted) superposition of the spectra corresponding to the respective distortions. If we take into account the finite width of $\left|\phi_{00}^{N_{e}}(\mathbf{Q})\right|^{2}$ it follows from Eq. (9) that the spectral features are broadened due to the Q-dependence of $V_{m}^{N_{e} \mp 1}(\mathbf{Q})-V_{0}^{N_{e}}(\mathbf{Q})$. We will consider a specific example in section 3 .

This analysis leads to our main conclusion. For the undoped system it follows from Eq. (5) that $\mathbf{Q}_{\text {min }}=0$. Consequently, the spectrum is just the broadened spectrum of the same system without EPI $\left(H_{e p}=0\right)$. The dispersion of the $\left(H_{e p}=0\right)$-quasi-particle peak shows up in the $\mathbf{k}$-dependence of the broadened peak in the low binding energy part of the spectra. This approach is particularly useful for the undoped system as it allows statements about the spectrum for $H_{e p} \neq 0$ from the knowledge of the spectrum for $H_{e p}=0$. For the doped system $\mathbf{Q}_{\min }$ is typically non-zero if the EPI is strong. The spectrum 
$\rho_{\mathbf{k}, \sigma}^{N_{e}, \mp}\left(\omega, \mathbf{Q}_{\min }\right)$ can then be very different from the spectrum for $\mathbf{Q}=0$. Therefore, even if the $\left(H_{e p}=0\right)$-spectrum is known, in general no information about the spectrum of the system with EPI can be deduced. In this context we notice that there may be several degenerate minima at $\mathbf{Q}_{\min } \neq 0$ such that the ground-state has an undistorted lattice in the sense of a vanishing expectation value of $\mathbf{Q}$. The spectrum for $H_{e p} \neq 0$, nevertheless, corresponds to a superposition of the spectra for $\mathbf{Q}$-values around the minima $\mathbf{Q}_{\min } \neq 0$.

The numerical calculation of spectral functions using the approximation in Eq. (9) can be quite efficient compared to other methods typically used to obtain spectra. In general, it is much easier to obtain the spectrum $\rho_{\mathbf{k}, \sigma}^{N_{e}, \mp}(\omega, \mathbf{Q})$ for a system without EPI but a given distortion $\mathbf{Q}$ than for a system with EPI. If the initial state's phonon wavefunction $\phi_{00}^{N_{e}}(\mathbf{Q})$ is known (e.g. in the case of an undoped system) its square can be used as a weight function in a Monte Carlo integration over the phonon coordinates in Eq. (9). The numerical effort is independent of the strength of the EPI. On the other hand, if e.g. exact diagonalization is used to obtain directly the ARPES spectrum of a system with EPI, calculations become computationally very demanding with increasing coupling strength as the truncated phonon Hilbert space grows larger and larger.

In order to improve the present approximation we have to include also the kinetic energy of the phonons in the resolvent of the Hamiltonian. Then, $\psi\left|E_{0}^{N_{e}}(\mathbf{Q})\right\rangle$ in Eq. (6) must be expanded with respect to both electronic and phononic basis functions in the adiabatic approximation. From this, one obtains the following expression for the spectral function:

$$
\begin{aligned}
& A_{\mathbf{k}, \sigma}^{N_{e}, \mp}(\omega)=\sum_{m, n} \delta\left(\omega-\left(\varepsilon_{m n}^{N_{e} \mp 1}-\varepsilon_{00}^{N_{e}}\right)\right) \times \\
& \quad \times\left|\int d \mathbf{Q}\left\langle E_{m}^{N_{e} \mp 1}(\mathbf{Q})|\psi| E_{0}^{N_{e}}(\mathbf{Q})\right\rangle \phi_{m n}^{N_{e} \mp 1}(\mathbf{Q}) \phi_{00}^{N_{e}}(\mathbf{Q})\right|^{2} .
\end{aligned}
$$

Each eigenstate of $H$ in the $\left(N_{e} \mp 1\right)$-electron sector represents a possible final state. It contributes to the spectrum at its eigenenergy (shifted by the ground-state energy of the system with $N_{e}$ electrons). The intensity is proportional to the squared overlap of final and initial state, i.e. the ground-state of the system with $N_{e}$ electrons plus an additional hole or electron. Two conditions must be fulfilled for this overlap to be large. ${ }^{1}$

i) As the initial phonon wavefunction $\phi_{00}^{N_{e}}(\mathbf{Q})$ is localized around the minimum $\mathbf{Q}_{\text {min }}$ of $V_{0}^{N_{e}}(\mathbf{Q})$ and has no nodes, the final phonon wavefunction $\phi_{m n}^{N_{e} \mp 1}(\mathbf{Q})$ must have a large and slowly varying amplitude in this region, too. This will be the case for final states with energies $\varepsilon_{m n}^{N_{e} \mp 1} \approx$ $V_{m}^{N_{e} \mp 1}\left(\mathbf{Q}_{\text {min }}\right)$. For smaller energies the region around $\mathbf{Q}_{m i}$ is classically forbidden and the amplitude of the final phonon wavefunction becomes exponentially suppressed, whereas for larger energies the kinetic energy increases and the

\footnotetext{
${ }^{1}$ In the following discussion we assume a non-degenerate minimum of $V_{0}^{N_{e}}(\mathbf{Q})$ for simplicity. The arguments can be easily generalized to the case of (quasi-)degenerate minima.
}

wavefunction oscillates faster. In both cases the integrated overlap of initial and final phonon wavefunction becomes smaller again.

ii) The electronic matrix element $\left\langle E_{m}^{N_{e} \mp 1}(\mathbf{Q})|\psi| E_{0}^{N_{e}}(\mathbf{Q})\right\rangle$ must be large around $\mathbf{Q}_{\text {min }}$. It is sufficient to consider its value only in this region as the initial phonon wavefunction is small elsewhere.

Altogether this leads to the following picture: For a system without EPI but with a given lattice distortion $\mathbf{Q}_{\text {min }}$ the spectrum consists of $\delta$-functions at the energies $E_{m}^{N_{e} \mp 1}\left(\mathbf{Q}_{\text {min }}\right)$. If the EPI is switched on, spectral features with large intensities will still appear at similar energies and with similar relative weight but they will be broadened by phonon sidebands. The quasiparticle's dispersion and weight, however, can be strongly altered by the EPI. In general, the effective phonon potential $V_{0}^{N_{e} \mp 1}(\mathbf{Q})$ corresponding to the electronic ground-state in the system with $N_{e} \mp 1$ electrons has minima at $\mathbf{Q} \neq \mathbf{Q}_{\text {min }}$. The ground-state phonon wavefunction $\phi_{00}^{N_{e} \mp 1}(\mathbf{Q})$ is localized around these minima. Consequently, there is only little overlap with the phonon wavefunction in the initial state which peaks around $\mathbf{Q}_{\min }$ and in the spectrum the peak lowest in binding energy has only very small weight.

It is interesting to discuss the problem above in terms of a sum-rule concerning the first moment (center of gravity) of the spectrum. For the undoped system with $N_{e}^{0}$ electrons that has no phonons excited in the initial state, one can show under rather general assumptions that the first moment of the (inverse) photoemission spectrum does not depend on the strength of the EPI. Therefore, the center of gravity of the spectrum does not change when the EPI is turned on. If in the absence of EPI $A_{\mathbf{k}, \sigma}^{N_{e}^{0}, \mp}(\omega)$ has only one peak for a given $\mathbf{k}$, e.g. if there is only one band and no electron-electron interaction, the center of gravity equals the position of the quasi-particle peak. Because of the sum-rule we then expect just a broadening but no shift of the quasi-particle peak upon switching on the EPI. In case of electrons interacting with themselves, however, already for systems without EPI $A_{\mathbf{k}, \sigma}^{N_{e}^{0} \text {, }}(\omega)$ usually has several peaks for a given $\mathbf{k}$ and the first moment does not correspond to the quasi-particle energy. Then, the sum-rule is not able to tell us how the individual peaks are influenced by the EPI and cannot be used to argue for prominent features in the spectrum dispersing approximately like the quasi-particles in the system without phonons.

\section{Holstein model}

In the following we demonstrate the validity of the arguments given above with several examples. First, we consider the one-dimensional $N$-site Holstein model with periodic boundary conditions for which the electronic part of the Hamiltonian just contains nearest-neighbor hopping with amplitude $t$ :

$$
H_{e l}=-2 t \sum_{k, \sigma} \cos (k) c_{k, \sigma}^{\dagger} c_{k, \sigma},
$$


where $c_{k, \sigma}^{\dagger}$ creates an electron with momentum $k$ and spin $\sigma$. The electrons are coupled to dispersionless phonons with frequency $\omega_{p h}$ as described by

$$
H_{e p}=\frac{g}{\sqrt{N}} \sum_{q, k, \sigma} \sqrt{2 \omega_{p h}} Q_{q} c_{k+q, \sigma}^{\dagger} c_{k, \sigma} .
$$

The strength of the interaction is given by $g$. The $(q=0)$ phonon mode couples to the total number of electrons, $N_{e}$. We can therefore write $H=H_{1}+H_{2}$, where

$$
H_{1}=N_{e} \frac{g}{\sqrt{N}} \sqrt{2 \omega_{p h}} Q_{0}+\frac{1}{2}\left(\Pi_{0}^{2}+\omega_{p h}^{2} Q_{0}^{2}\right)
$$

can be solved exactly [9]. The spectral function $A_{k, \sigma}^{N_{e}, \mp(2)}(\omega)$ obtained for $H_{2}$ only needs to be convoluted by the known result for $H_{1}$,

$$
A^{N_{e}, \mp(1)}(\omega)=e^{-\alpha} \sum_{l=0}^{\infty} \frac{\alpha^{l}}{l !} \delta\left(\omega+\left(1 \mp 2 N_{e}\right) \alpha \omega_{p h}-l \omega_{p h}\right),
$$

where $\alpha=\left(g / \omega_{p h}\right)^{2} / N$, in order to get $A_{k, \sigma}^{N_{e}, \mp}(\omega)$ as defined in Eq. (11). We will therefore restrict our discussion to $A_{k, \sigma}^{N_{e}, \mp(2)}(\omega)$ in the following.

We specifically consider a two-site system $(N=2)$ [13] and calculate the inverse photoemission spectrum for creating an electron in both the empty (undoped) system and the system that already contains an electron of opposite spin. There is then only one phonon coordinate $Q_{\pi}$ in $\mathrm{H}_{2}$ which we treat as a parameter in solving the part of $\mathrm{H}_{2}$ coming from $\mathrm{H}_{e l}+H_{e p}$. In the one-electron sector one obtains the two eigenenergies

$$
E_{0 / 1}^{N_{e}=1}=\mp \sqrt{t^{2}+\omega_{p h} g^{2} Q_{\pi}^{2}},
$$

whereas there are four eigenergies in case of two electrons with opposite spin:

$$
E_{0 / 3}^{N_{e}=2}=\mp 2 \sqrt{t^{2}+\omega_{p h} g^{2} Q_{\pi}^{2}}, \quad E_{1 / 2}^{N_{e}=2}=0 .
$$

For numerical calculations we choose $t=1, \omega_{p h}=0.1$, and $g=0.6$. As $\omega_{p h} / t$ is small our adiabatic approximation is justified. The effective potentials one obtains by adding the harmonic potential $\omega_{p h}^{2} Q_{\pi}^{2} / 2$ to the eigenenergies in Eqs. (16) are shown in Fig. 1.

We first consider the approximation when the kinetic energy of the phonons is neglected in the resolvent of the Hamiltonian and for which the spectral function is given by Eq. (9). The neglected terms are proportional to $\omega_{p h}$ so we cannot expect to resolve fine-structure in the spectra on that order. But it turns out that this approximation still describes the overall broadening correctly on a larger scale proportional to $\sqrt{\omega_{p h}}[12]$. The phonon wavefunction in the initial state is known exactly for the undoped system , see Eq. (5). Here

$$
\phi_{00}^{N_{e}^{0}=0}\left(Q_{\pi}\right)=\left(\omega_{p h} / \pi\right)^{(1 / 4)} \exp \left(-\omega_{p h} Q_{\pi}^{2} / 2\right) .
$$

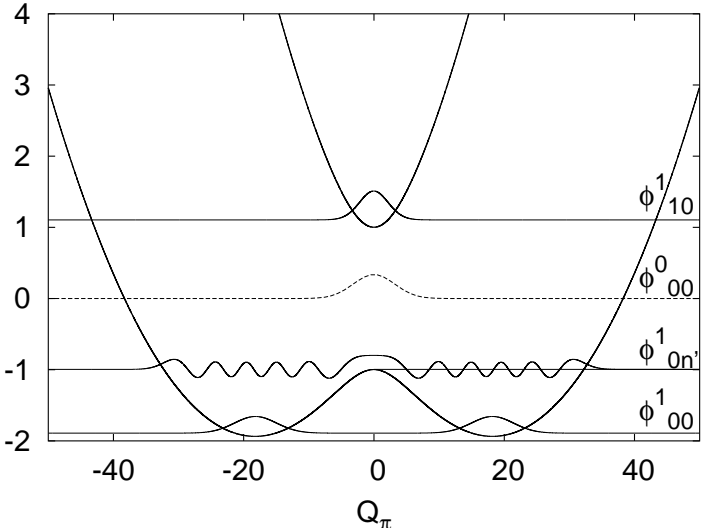

Fig. 1. Effective potentials $V_{m}^{1}\left(Q_{\pi}\right)$ in the two-site Holstein model with one electron as functions of the phonon coordinate $Q_{\pi}$ for $t=1, \omega_{p h}=0.1, g=0.6$. Some selected phonon wavefunctions are also shown, see text.

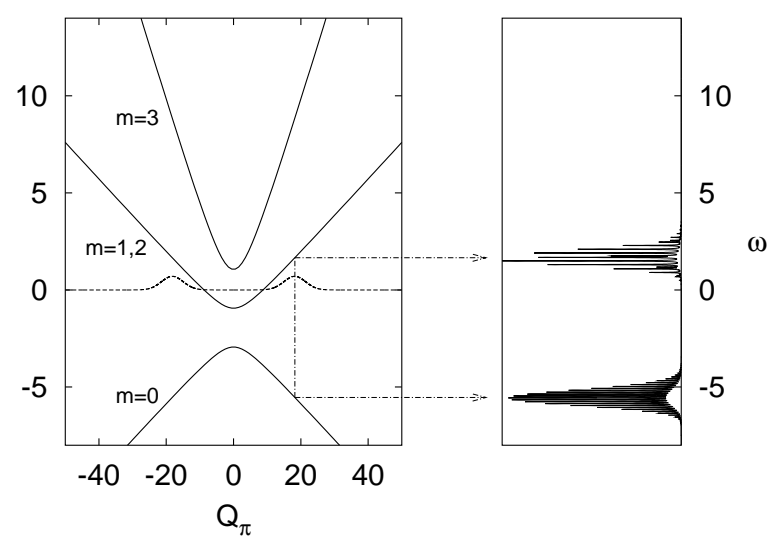

Fig. 2. Left panel: Effective potentials $V_{m}^{2}\left(Q_{\pi}\right)-V_{0}^{1}\left(Q_{\pi}\right)+$ $\left.V_{0}^{1}\left(Q_{\pi}\right)\right|_{\bar{Q}_{ \pm}}$for the two-site Holstein model with two electrons with opposite spin as functions of the phonon coordinate $Q_{\pi}$ for $t=1, \omega_{p h}=0.1, g=0.6$ and phonon wavefunction $\phi_{00}^{N_{e}=1}$ (dotted line). Right panel: $A_{k=0, \sigma}^{N_{e}=1,+(2)}(\omega)$ rotated by $90^{\circ}$.

In the system with one electron the lowest effective potential $V_{0}^{N_{e}=1}\left(Q_{\pi}\right)$ has two minima at $\bar{Q}_{ \pm}$around which we can treat it as harmonic potential with renormalized phonon frequency $\bar{\omega}=\sqrt{\left.\left(\partial^{2} V_{0}^{N_{e}=1} / \partial Q_{\pi}^{2}\right)\right|_{\bar{Q}_{ \pm}}}$. This leads to the approximation

$$
\phi_{00}^{N_{e}=1}\left(Q_{\pi}\right) \approx \frac{1}{\sqrt{2}} \sum_{i= \pm}\left(\frac{\bar{\omega}}{\pi}\right)^{1 / 4} \exp \left(-\bar{\omega}\left(Q_{\pi}-\bar{Q}_{i}\right)^{2} / 2\right)
$$

Following Ref. [12], we can expand the argument of the $\delta$-functions in Eq. (9) up to first order in $Q_{\pi}$ around $\bar{Q}_{ \pm}$. If we further assume that the electronic matrix elements vary only weakly around $\bar{Q}_{ \pm}$the integration over $Q_{\pi}$ in Eq. (9) can be eliminated and we obtain the following 


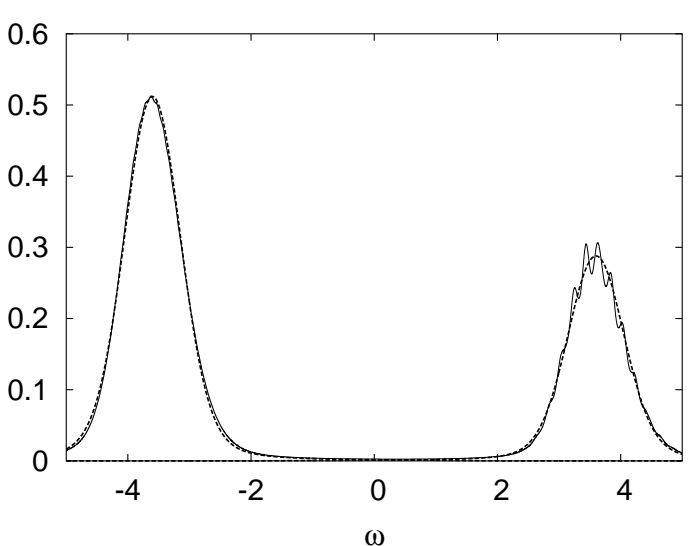

Fig. 3. Spectrum for adding an electron to a two-site Holstein model with one electron of opposite $\operatorname{spin}\left(t=1, \omega_{p h}=0.1\right.$, $g=0.6)$. The approximation $\tilde{A}_{k=0, \sigma}^{N_{e}=1,+(2)}(\omega)($ Eq. $(20))$ is shown as dashed line together with the spectrum obtained from exact diagonalization (thin line). Both spectra have been convoluted with a Lorentzian (FWHM=0.1).

result:

$\left.\tilde{A}_{k, \sigma}^{N_{e}=1,+(2)}(\omega) \approx \sum_{m}\left|\left\langle E_{m}^{2}\left|c_{k, \sigma}^{\dagger}\right| E_{0}^{1}\right\rangle\right|^{2}\right|_{\bar{Q}_{ \pm}} \sqrt{\frac{\bar{\omega}}{\pi b_{m}^{2}}} e^{-\frac{\bar{\omega}}{b_{m}^{2}}\left(\omega-a_{m}\right)^{2}}$

where $a_{m}=\left.V_{m}^{2}\right|_{\bar{Q}_{ \pm}}-\left.V_{0}^{1}\right|_{\bar{Q}_{ \pm}}$and $b_{m}=\left.\left(\partial V_{m}^{2} / \partial Q_{\pi}\right)\right|_{\bar{Q}_{ \pm}}$ This approximation is shown in Fig. 3 for $k=0$ together with the spectrum obtained from exact diagonalization. The agreement with the Gaussian lineshape predicted by Eq. (20) is very good. Only to resolve the fine-structure on a scale given by the phonon frequency $\omega_{p h}$ one has to go beyond the present approximation. As indicated schematically by the arrows in Fig. 2 the spectrum can indeed be understood as the spectrum of electrons in a system without EPI but a given distortion $\bar{Q}_{ \pm}$. The broadening is due to the finite width of the phonon wavefunction in the initial state. There is no structure in the spectrum arising from the highest effective potential as the corresponding electronic matrix element is negligible.

In the case of inverse photoemission from the empty system the phonon wavefunction in the initial state is localized around $Q_{\pi}=0$. The slope of both effective potentials $V_{m=0,1}^{N_{e}=1}\left(Q_{\pi}\right)$ vanishes at this point. An approximation analogous to Eq. (20) would therefore result in $\tilde{A}_{k, \sigma}^{N_{e}^{0}=0,+(2)}(\omega)=\rho_{k, \sigma}^{N_{e}^{0}=0,+}\left(\omega, Q_{\pi}=0\right)$, i.e. the unbroadened spectrum of the undistorted system without EPI. If we evaluate Eq. (9) without any further approximation we obtain the spectra shown in Fig. 4. The comparison with results from exact diagonalization shows that this approximation cannot reproduce the fine-structure and for $k=0$ does not give spectral weight above $\omega=-1$ (except from broadening), but it still gives the right order for the broadening of the peaks.

In order to understand also details of the spectra we have to use Eq. (11) which results from making no approximation other than the initial adiabatic one. One has

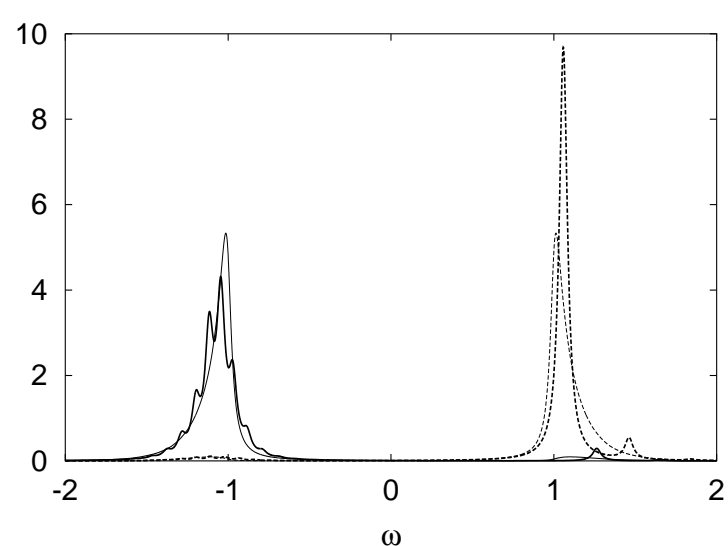

Fig. 4. Spectra for adding an electron to an empty two-site Holstein model $\left(t=1, \omega_{p h}=0.1, g=0.6\right)$. The approximation $\tilde{A}_{k, \sigma}^{N_{e}^{0}=0,+(2)}(\omega)$ (Eq. (9), thin lines) and results from exact diagonalization (bold lines) are shown for both $k=0$ (solid lines) and $k=\pi$ (dashed lines). All spectra include a Lorentzian broadening $(\mathrm{FHWM}=0.06)$.

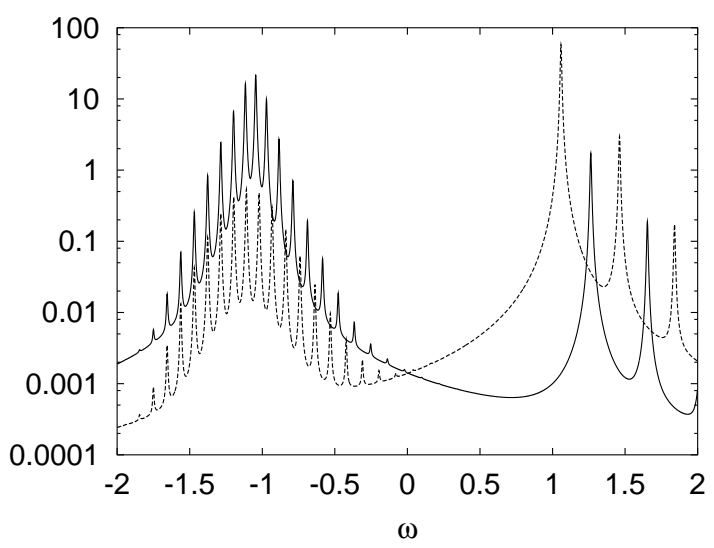

Fig. 5. Spectra $A_{k, \sigma}^{N_{e}^{0}=0,+(2)}(\omega)$ for adding an electron to an empty two-site Holstein model $\left(t=1, \omega_{p h}=0.1, g=0.6\right)$ as given by Eq. (11). The spectra for both $k=0$ (solid line) and $k=\pi$ (dashed line) are shown with Lorentzian broadening $(\mathrm{FWHM}=0.01)$. Observe the logarithmic intensity scale.

to solve Eq. (3) for each effective potential to obtain the phonon eigenfunctions needed in Eq. (11). The resulting inverse photoemission spectra for creating an electron with momentum $k=0$ or $k=\pi$ in the empty system are shown in Fig. 5. They are practically indistinguishable from those we obtained using exact diagonalization. This shows that the adiabatic approximation works very well for the chosen parameters. For the electronic matrix element in Eq. (11) one needs the $Q_{\pi}$-dependent electronic eigenstates corresponding to the eigenenergies in Eq. (16):

$\left|E_{0 / 1}^{N_{e}=1}\right\rangle=\mathcal{N}_{0 / 1}\left(\left(t \pm \sqrt{t^{2}+\omega_{p h} g^{2} Q_{\pi}^{2}}\right)|0\rangle-\sqrt{\omega_{p h}} g Q_{\pi}|\pi\rangle\right)$ 
with

$$
\mathcal{N}_{m}=\frac{\left(\operatorname{sign}\left(Q_{\pi}\right)\right)^{m}}{\sqrt{\left(t+(-1)^{m} \sqrt{\left.t^{2}+\omega_{p h} g^{2} Q_{\pi}^{2}\right)^{2}+\omega_{p h} g^{2} Q_{\pi}^{2}}\right.}}
$$

where $|0\rangle$ and $|\pi\rangle$ denote the $k=0$ and $k=\pi$ one-electron states and $m=0,1$.

Let us consider e.g. the case where we create an electron with momentum $k=0$ in the empty system. The initial electronic state is then $|0\rangle$. The initial state's phonon wavefunction $\phi_{00}^{N_{e}^{0}=0}$ (dashed line in Fig. 1) is a bell-shaped Gaussian centered around $Q_{\pi}=0$. This effectively limits the integration over $Q_{\pi}$ in Eq. (11) to a small region around the origin. Here $\left|E_{0}^{N_{e}=1}\right\rangle \approx|0\rangle$ and $\left|E_{1}^{N_{e}=1}\right\rangle \approx|\pi\rangle$. Therefore, the relevant electronic matrix element in this example is $\left\langle E_{0}^{N_{e}=1} \mid 0\right\rangle$. It has even parity with respect to $Q_{\pi}$. So, according to Eq. (11) final states with the electronic configuration $\left|E_{0}^{N_{e}=1}\right\rangle$ and a corresponding evenparity phonon wavefunction $\phi_{0 n}^{N_{e}=1}$ that strongly overlaps with $\phi_{00}^{N_{e}^{0}=0}$ give rise to large spectral intensity. We show in Fig. 1 the even-parity phonon wavefunction $\phi_{0 n^{\prime}}^{N_{e}=1}$ with the largest overlap offset along the ordinate by its eigenergy. It has a sizable and slowly varying amplitude around $Q_{\pi}=0$ because its eigenergy is close to the local value of the effective potential which at $Q_{\pi}=0$ equals the $(k=0)$-eigenenergy $-t$ of the system without EPI. Therefore, the peak with largest weight appears around this energy in the spectrum (solid line in Fig. 5). The sidepeaks arise from final states with even-parity phonon wavefunctions with lower or higher eigenenergies whose overlap with $\phi_{00}^{N_{e}^{0}=0}$ decreases. Figure 1 also shows the even parity ground-state phonon wavefunction $\phi_{00}^{N_{e}=1}$ in the doublewell potential. Clearly, its overlap with $\phi_{00}^{N_{e}^{0}=0}$ is very small leading to a strongly suppressed quasi-particle peak in $A_{k=0, \sigma}^{N_{e}^{0}=0,+(2)}$. Because of the large dimensionless EPI constant $\lambda=g^{2} /\left(\omega_{p h} t\right)=3.6$ we are well in the polaronic regime.

If, on the other hand, an electron with momentum $k=$ $\pi$ is created in the empty state one finds using similar arguments as before that final states leading to a large spectral intensity must have the electronic configuration $\left|E_{1}^{N_{e}=1}\right\rangle$. Their phonon wavefunction $\phi_{1 n}^{N_{e}=1}$ must strongly overlap with $\phi_{00}^{N_{e}^{0}=0}$ and be of even-parity. In this case the lowest energy phonon wavefunction $\phi_{10}^{N_{e}=1}$ in the upper effective potential (shown in Fig. 1) has the largest overlap because its eigenenergy is closest to $E_{1}^{N_{e}=1}\left(Q_{\pi}=0\right)=+t$. Therefore, $A_{k=0, \sigma}^{N_{e}^{0}=0,+(2)}(\omega)$ (dashed line in Fig. 5) shows a prominent peak at $\omega \approx+t$.

As $\left\langle E_{1}^{N_{e}=1} \mid 0\right\rangle\left(\left\langle E_{0}^{N_{e}=1} \mid \pi\right\rangle\right)$ only vanishes completely at $Q_{\pi}=0$ the spectrum for $k=0(k=\pi)$ also shows weak structures around $\omega=+t(\omega=-t)$ where the coupling is now to phonon wavefunctions of odd parity. The density of coupling phonon states is different around $\omega=-t$ and $\omega=$ $+t$. E.g., the fact that the upper effective potential has a minimum around $Q_{\pi}=0$ results in an asymmetric shape

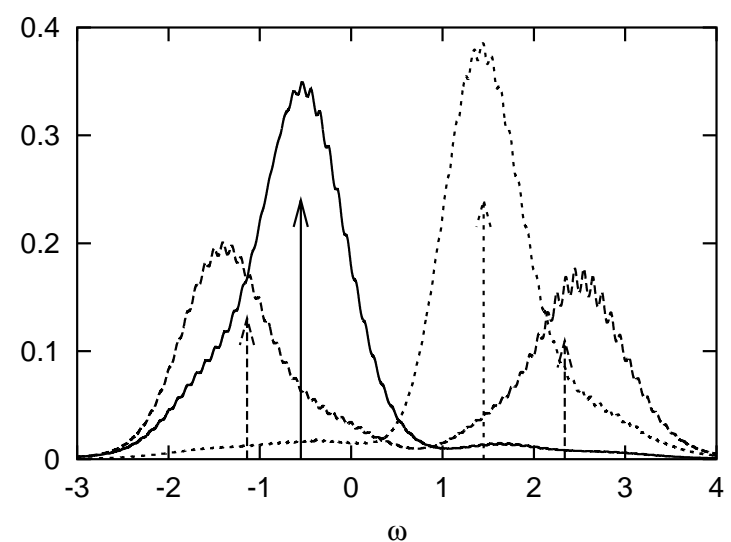

Fig. 6. Spectral functions $A_{k, \sigma}^{N_{h}^{0}=0,-}(\omega)$ for creating a hole in the undoped 4-site Holstein- $t-J$ model $\left(t=1, J=0.3, \omega_{p h}=\right.$ $0.1, g=0.8) . k=0$ : solid line, $k= \pm \pi / 2$ : dashed line, $k=$ $\pi$ : dotted line (Lorentzian broadening: FWHM=0.01). Arrows show positions and weights of corresponding peaks for $g=0$.

of $A_{k, \sigma}^{N_{e}^{0},+(2)}(\omega)$ around $\omega=+t$ as no phonon eigenstates in this effective potential can have eigenenergies below $+t$.

\section{$4 t-J$ model with phonons}

As a second example we study the one-dimensional $N$-site Holstein- $t-J$ model with periodic boundary conditions. This model also includes electron-electron interactions. The electronic part of $H$ is given by the usual $t-J$ Hamiltonian

$$
\begin{aligned}
H_{e l}=- & t \sum_{i, \sigma}\left(\tilde{c}_{i, \sigma}^{\dagger} \tilde{c}_{i+1, \sigma}+\text { h.c. }\right) \\
& +J \sum_{i}\left(\mathbf{S}_{i} \cdot \mathbf{S}_{i+1}-\frac{n_{i} n_{i+1}}{4}\right),
\end{aligned}
$$

where $\tilde{c}_{i, \sigma}^{\dagger}$ creates an electron with spin $\sigma$ on site $i$ if this site was previously empty, $n_{i}=\sum_{\sigma} \tilde{c}_{i, \sigma}^{\dagger} \tilde{c}_{i, \sigma}$, and $\mathbf{S}_{i}$ is a spin- $\frac{1}{2}$ operator. Besides the hopping $t$ there is also an exchange coupling parameter $J$. As in the Holstein-model we consider an interaction with dispersionless phonons where the coupling is now to empty sites (holes):

$$
H_{e p}=\frac{g}{\sqrt{N}} \sum_{q, j} \sqrt{2 \omega_{p h}} Q_{q}\left(1-n_{j}\right) e^{i q j}
$$

The system with one electron per site corresponds to the undoped case where the EPI vanishes. The $(q=0)$-phonon mode can be treated separately again, the only difference being that the coupling is now proportional to the total number of empty sites $N_{h}$, not to the total number of electrons $N_{e}$.

For numerical calculations we consider a 4-site system with $t=1, J=0.3, \omega_{p h}=0.1$, and $g=0.8$. The photoemission spectra for destroying an electron with momentum $k$ and spin $\sigma$ were obtained using exact diagonalization with up to 200 phonons per basis state for solving the 


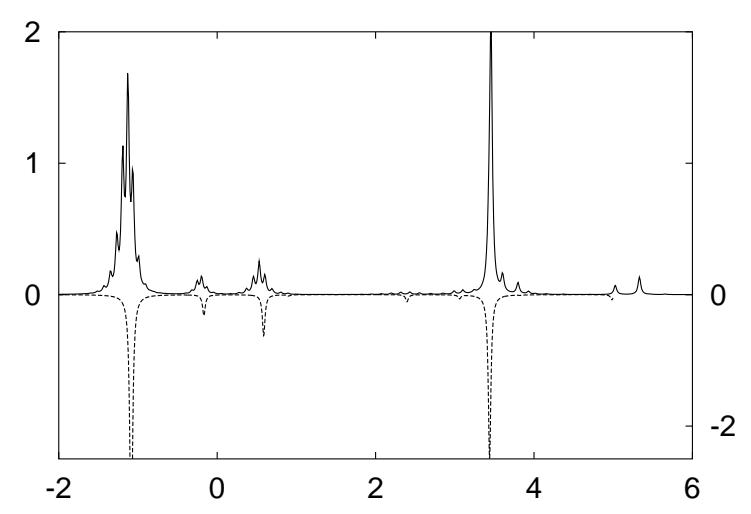

(a)

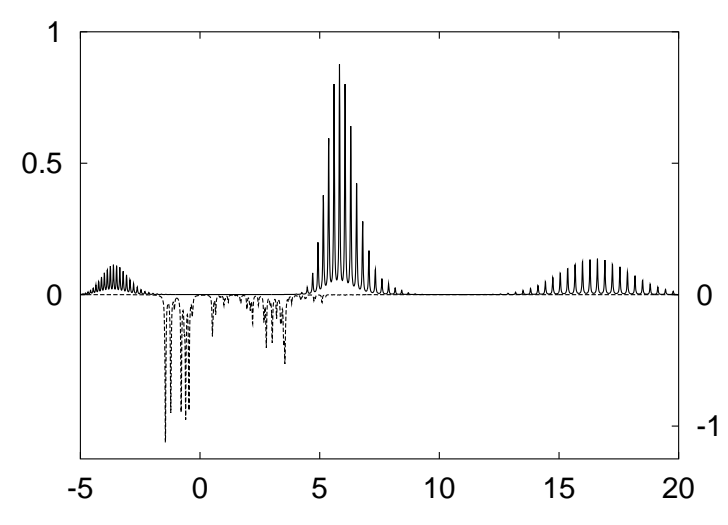

$\omega$

Fig. 7. Inverse photoemission spectra $(\mathbf{k}=(3,1) \pi / 5)$ for the 10 -site $t$ - $J$ model with one interacting phonon mode $(t=1$, $J=0.4, \omega_{p h}=0.1, g_{(\pi, \pi)}=1.6$ ): (a) from the undoped system, (b) from the 10\%-doped system. The corresponding spectra for systems without EPI are shown by dashed lines with their amplitude flipped for clarity. A Lorentzian broadening of FWHM=0.04 has been applied.

problem without the $(q=0)$-mode and subsequent convolution with $A^{N_{h}^{0}=0,-(1)}(\omega)$ (Eq. (15) with $N_{h}=0$ instead of $N_{e}$ ). The results are displayed in Fig. 6 together with arrows indicating the peaks in the corresponding spectra for $g=0$. Without EPI there is only one peak both for $k=0$ (at -0.55$)$ as well as for $k=\pi$ (at 1.45). For $k= \pm \pi / 2$ the spectrum has two peaks at -1.139 and 2.339 as a result of the electron-electron interaction.

Again, the dispersion found in the system without EPI is traced quite accurately by a broad peak in the case of strong EPI. The spectrum for $k= \pm \pi / 2$ also illustrates our comments at the end of section 2 on the sum-rule concerning the first spectral moment. Since the spectrum has two peaks for $k= \pm \pi / 2$, the sum rule cannot tell us how the peaks are broadened. Many other spectra would also have been consistent with the sum-rule, e.g. spectra where the peaks are shifted. The arguments based on the adiabatic approximation, however, show that both peaks should be broadened with their individual center of gravity remaining roughly unchanged in agreement with the exact calculations.

Finally, we consider the $t-J$ model in two dimensions on a tilted 10-site cluster with periodic boundary conditions. To simplify calculations we assume that the EPI is described by Eq. (24) but with a q-dependent coupling constant $g_{\mathbf{q}}$. In the following we choose $g_{(\pi, \pi)}=1.6$ and $g_{\mathbf{q}}=0$ for all other $\mathbf{q} \neq(\pi, \pi)$ so that effectively there is only one phonon mode that interacts with the electrons. The other parameters are $t=1, J=0.4$, and $\omega_{p h}=0.1$. Figure 7 shows the inverse photoemission spectra for $\mathbf{k}=(3,1) \pi / 5$ from both the undoped (Fig. $7(\mathrm{a})$ ) and the $10 \%$-doped system (Fig. 7(b)). The EPI has been switched on and off (solid line vs. dashed line with flipped amplitude).

The spectra from the undoped system confirm again our general expectations from section 2. The EPI basically broadens the structures in the original spectrum. This includes the quasi-particle peak at low binding energies. In contrast, the spectrum from the doped system changes quite differently when the EPI is switched on. Although the spectrum again develops several broad features they cannot be related anymore in a simple way to the structures in the spectrum found without EPI. We have also varied $\mathbf{k}$ and found that the dispersion of the broad features is different from the quasi-particle dispersion in the system without EPI. According to Eq. (9) the spectra rather correspond to broadened versions of spectra one would obtain in a purely electronic, but distorted system.

\section{Conclusions}

We have introduced an adiabatic approximation for calculating ARPES spectra from systems with strong coupling of doped carriers to phonons. The effective phonon potential for the initial state is calculated as a function of the phonon coordinates and its minima are found. We show that the spectrum with electron-phonon interaction (EPI) is then related to a broadened average of spectra without EPI calculated for distorted lattices corresponding to the minima of the effective potential. We also studied the additional approximation of neglecting the kinetic energy of the phonons in the resolvent of the Hamiltonian corresponding to the ARPES Green's function. The spectrum is then expressed as superposition of spectra from distorted lattices without EPI, using the square of the initial state's phonon wave function as a weight function (see Eq. (9)).

In either form, the theory becomes particularly simple if the EPI can be neglected in the initial state. The phonon wave function is then centered around the undistorted lattice, and the spectrum with EPI can be directly related to the (broadened) spectrum without EPI. In the case of strong EPI in the initial state, the minima of the effective potential correspond to distorted lattices. The spectrum with EPI is then related to the (broadened) spectra of distorted lattices without EPI. Therefore, the knowledge of the spectrum without EPI for the undistorted lattice is in 
general not very informative with respect to the spectrum with EPI.

Our results support the interpretation of ARPES on undoped high- $T_{c}$ cuprates $[6,7]$ and explain why in numerical calculations [8] the quasi-particle dispersion from purely electronic models shows up almost unchanged in the dispersion of incoherent features in the spectra obtained with EPI.

\section{References}

1. L. Perfetti, S. Mitrovic, G. Margaritondo, M. Grioni, L. Forró, L. Degiorgi, and H. Höchst, Phys. Rev. B 66, 075107 (2002)

2. L. Perfetti, H. Berger, L. Degiorgi, H. Höchst, J. Voit, G. Margaritondo, and M. Grioni, Phys. Rev. Lett. 87, 216404 (2001)

3. D. S. Dessau, T. Saitoh, C.-H. Park, Z.-X. Shen, P. Villella, N. Hamada, Y. Moritomo, and Y. Tokura, Phys. Rev. Lett. 81, $192(1998)$

4. T. Tohyama and S. Maekawa, Supercond. Sci. Technol. 13, R17 (2000)

5. A. Damascelli, Z. Hussain, and Z.-X. Shen, Rev. Mod. Phys. 75, 473

6. K. M. Shen et al., cond-mat/0407002 (2004)

7. K. M. Shen et al., unpublished

8. A.S. Mishchenko and N. Nagaosa, Phys. Rev. Lett. 93 , $036402(2004)$

9. G. D. Mahan, Many-Particle Physics, $3^{\text {rd }}$ edition (Kluwer Academic/Plenum Publishers, New York, 2000), p. 218

10. V. Perebeinos and P. B. Allen, Phys. Rev. Lett. 85, 5178 (2000)

11. M. Born and K. Huang, Dynamical Theory of Crystal Lattices (Oxford University Press, London, 1954)

12. K. Schönhammer and O. Gunnarsson, Phys. Rev. B 30 , 3141 (1984)

13. J. Ranninger and U. Thibblin, Phys. Rev. B 45, 7730 (1992) 